\title{
Identification and characterization of ZL261, a novel Collimonas pratensis strain with antagonistic activity toward Monilinia fructicola
}

\author{
Huiling Wu, Jieqiong Li, Weicheng Liu*, Xuelian Bai, Dewen Liu \& Jiewei Zhang \\ Institute of Plant and Environment Protection, Beijing Academy of Agriculture and Forestry Sciences, Beijing 100097, China
}

Received July 11, 2016; August 17, 2016; published online October 21, 2016

Citation: $\quad$ Wu, H., Li, J., Liu, W., Bai, X., Liu, D. and Zhang, J. (2016). Identification and characterization of ZL261, a novel Collimonas pratensis strain with antagonistic activity toward Monilinia fructicola. Sci China Life Sci 59, 1345-1347. doi: 10.1007/s11427-016-0066-9

\section{Dear Editor,}

Soil bacteria from the genus Collimonas were isolated for the first time by De Boer et al., and they have been shown to display antifungal activity toward many different fungi (De Boer et al., 1998). These affected fungi include pathogens of economically important plants, such as spruce, potato, flax, and lettuce, suggesting the potential for the broad application of Collimonas as a biocontrol agent. Brown rot caused by Monilinia fructicola is among the most important postharvest diseases of commercially grown stone fruits in production areas worldwide (Pellegrino et al., 2009). Application of chemical fungicides is currently the most common method to control brown rot. However, chemical controls can leave fungicide residue on the fruits and induce fungicide resistance in the pathogen. The biological control of plant diseases by using antagonistic microorganisms is a promising strategy for sustainable agriculture. Strain ZL261 isolated from alpine meadow soil on Sejila Mountain in Tibet, China, exhibited a specific inhibitory effect against the brown rot pathogen $M$. fructicola. The sequence of the $16 \mathrm{~S}$ rDNA gene in ZL261 showed 99\% similarity with those in Collimonas pratensis, C. fungivorans, and C. arenae. The DNA-DNA hybridization value between strain ZL261 and C. pratensis strain CCUG $54728 \mathrm{~T}$ was $83.88 \%$, suggesting

*Corresponding author (email: liuwich@163.com) that ZL261 belonged to the species $C$. pratensis.

Physiological and biochemical analyses revealed that strain ZL261 was an aerobic, gram-negative bacterium and that it could produce purple pigment on KB medium. Analysis of the utilization of 21 carbon sources by strain ZL261 and three other strains belonging to different Collimonas spp. indicated that there were significant differences in carbon source utilization by strain ZL261 and the three other Collimonas strains (Table S1 in Supporting Information).

No antagonistic activity was found for strain ZL261 against any of the tested bacteria and most of the tested plant pathogenic fungi using the dual culture assay on PDA plates at $25^{\circ} \mathrm{C}$. However, M. fructicola, the causal agent of brown rot on stone fruits, was greatly inhibited by ZL261. The mean diameter of the M. fructicola colonies co-cultured with strain ZL261 for seven days was only $2 \mathrm{~cm}$, which was significantly smaller than those co-cultured with C. pratensis CCUG $54728^{\mathrm{T}}(5.2 \mathrm{~cm})$, C. fungivorans CCUG $48868^{\mathrm{T}}$ $(3.7 \mathrm{~cm})$, and $C$. arenae CCUG $54727^{\mathrm{T}}(4.1 \mathrm{~cm})$, as well as the control $(7.2 \mathrm{~cm})$ (Table $\mathrm{S} 2$ in Supporting Information). When the dual culture plates were incubated for another 10 days, the $M$. fructicola colony confined between the two streaks of strain ZL261 was still much smaller than those in the control and other treatment groups, which indicated a persistent inhibition effect of ZL261 (Figure S1 in Supporting Information). Meanwhile, all three reference strains of Collimonas spp. were overwhelmed by M. fructicola (Figure 
S2 in Supporting Information).

The results of Leveau et al. demonstrated that differences in the susceptibility of individual fungi to different Collimonas strains existed, whereas individual Collimonas strains differed in the types of fungi that they inhibited, as well as in the extent of the inhibition (Leveau et al., 2010). For example, only the C. fungivorans strain Ter331 among the Collimonas strains was reported to show antagonistic activity against tomato foot and root rot caused by Fusarium oxysporum f. sp. radicis-lycopersici. Meanwhile, M. hiemalis and $P$. exigua were affected at least somewhat by all of the Collimonas strains except Ter330. Other groups reported the antifungal activity of the Collimonas strain IS343 against Rhizoctonia solani, as well as the antifungal activity of the volatiles produced by $C$. arenae Ter10 and $C$. fungivorans Ter331 against Chaetomium sp. However, Chaetomium sp. was not affected by C. pratensis Ter 91 (Garbeva et al., 2014).

The antagonistic activity of strain ZL261 on the spore germination and mycelial growth of $M$. fructicola was qualitatively determined by the agar well diffusion method.

ZL261 was cultured for $36 \mathrm{~h}$ in KB medium, and the culture supernatant was then centrifuged at $10,000 \times \mathrm{g}$ for $15 \mathrm{~min}$ and used for the antagonistic activity test. After incubation for three days, the edges of the colonies were cut with a sterile scalpel and observed under a light microscope (Leica DMIRE2 microscope, Germany), and each treatment was performed in triplicate.

The average diameter of the $M$. fructicola-inhibition zones produced by the fermentation supernatant of ZL261 was $(2.3 \pm 0.06) \mathrm{cm}$, whereas the control treatment had no inhibition effect on M. fructicola (Figure S3A in Supporting Information). Microscopy analysis revealed differences in sporulation and hyphal morphology between M. fructicola treated with the fermentation supernatant of strain ZL261 and M. fructicola treated with sterile water. When treated with the fermentation supernatant of strain ZL261, the spore germination of M. fructicola was significantly inhibited, and almost no mature spores were formed. The spores of $M$. fructicola without treatment with strain ZL261 germinated very well and were plump in shape (Figure S3B in Supporting Information). In addition, the hyphae of $M$. fructicola without treatment with strain ZL261 were dense, plump shaped, and regularly arranged (Figure S3C in Supporting Information). On the contrary, M. fructicola treated with the fermentation supernatant of strain ZL261 had some morphological abnormalities, such as an irregular arrangement and breaks.

To evaluate the antagonistic activity of strain ZL261 against peach brown rot, ripe peaches (cv. Springcrest) from conventional production were surface-disinfected with $0.5 \%$ commercial sodium hypochlorite for one minute and then rinsed with sterilized tap water. Three artificial wounds (approximately $3 \mathrm{~mm}$ wide $\times 3 \mathrm{~mm}$ deep) were made along the equa- torial zone of the peaches, and $30 \mu \mathrm{L}$ fermentation broth of strain ZL261 was added into each wound. After air-drying for two hours at $25^{\circ} \mathrm{C}$, a $50 \mu \mathrm{L}$ aliquot of a $M$. fructicola suspension at $1 \times 10^{5}$ conidia $\mathrm{mL}^{-1}$ was pipetted into each wound. Fruits treated with only the pathogen $M$. fructicola or only the fermentation broth of strain ZL261 served as controls. Additionally, fruits treated with the fungicide carbendazim and sterile distilled water were used as the chemical and the blank controls, respectively. All of the fruits were incubated at $25^{\circ} \mathrm{C}$ with $95 \% \mathrm{RH}$. Six days after the inoculation, the disease incidence (percentage of infected wounds) was recorded, and the diameters of the lesions were measured. Each treatment was replicated three times, with 12 fruits per treatment, and the experiment was repeated three times.

The results showed that strain ZL261 significantly inhibited the development of the brown rot caused by M. fructicola on peaches. After 6 days, all the peaches inoculated with $M$. fructicola were infected, and the average diameter of the brown rot lesions was $32.7 \mathrm{~mm}$, whereas the peaches treated with strain ZL261 before $M$. fructicola inoculation had a low disease incidence of $17.8 \%$ and an average brown rot lesion diameter of $8.9 \mathrm{~mm}$ (Table 1). The brown rot lesion diameter and disease incidence in the carbendazim treatment group were $6.8 \mathrm{~mm}$ and $9.8 \%$, respectively, whereas the fruits treated with sterile distilled water or the ZL261 control had no disease symptoms (Table 1). Compared to the carbendazim treatment, treatment with strain ZL261 displayed a similar effect on the control of peach brown rot, with a slightly higher disease incidence.

Several previous studies have investigated the mechanisms of fungal suppression by collimonads. For example, chitinase activity was demonstrated by De Boer et al. to be the antagonistic factor (De Boer et al., 1998), and Collimonas Ter331 suppressed $F$. oxysporum on tomato roots by inhibiting its hyphae (Kamilova et al., 2007). Investigations regarding the interactions of Collimonas sp. Ter331 with $F$. oxysporum and Collimonas sp. IS343 with $R$. solani revealed that pathogenic fungi suppression was not due to antibiotic activity or fungal cell wall degradation, but competition for available nutrients

Table 1 Efficacy of the strain ZL261 on controlling peach brown rot on stone fruit caused by $M$. fructicola ${ }^{\text {a) }}$

\begin{tabular}{ccc}
\hline Treatment & Disease incidence (\%) & $\begin{array}{c}\text { Diameter of brown } \\
\text { rot lesion }(\mathrm{mm})^{*}\end{array}$ \\
\hline ZL261 & $17.8 \pm 2.3 \mathrm{~b}$ & $8.9 \pm 2.2 \mathrm{~b}$ \\
Inoculated control & $100 \pm 0.0 \mathrm{a}$ & $32.7 \pm 3.6 \mathrm{a}$ \\
Carbendazim & $9.8 \pm 1.6 \mathrm{c}$ & $6.8 \pm 1.2 \mathrm{~b}$ \\
ZL261 control & $0 \pm 0.0 \mathrm{~d}$ & $0 \pm 0.0 \mathrm{c}$ \\
Blank control & $0 \pm 0.0 \mathrm{~d}$ & $0 \pm 0.0 \mathrm{c}$ \\
\hline
\end{tabular}

a) *, The results are the mean of three independent experiments. " \pm " stands for standard deviation of the mean. Values followed by the same letter are not statistically different by Duncan's multiple range test $(P<0.05)$. 
(Kamilova et al., 2007; Leveau et al., 2010). These previous results demonstrated that there are various mechanisms for the suppression of pathogenic fungi by Collimonas strains, and the common phenomenon of these mechanisms was a reduction in the mycelium density of the pathogens. Our preliminary observation of the inhibition of M. fructicola spore germination and mycelial growth by strain ZL261 could further accelerate the elucidation of the exact antifungal mechanisms of Collimonas.

In conclusion, in this study, strain ZL261 isolated from alpine meadow soil on Sejila Mountain, Tibet, China, was identified as a novel strain of $C$. pratensis by using physiological, biochemical, and molecular methods. ZL261 exhibited a specific inhibitory effect against $M$. fructicola, the causal agent of brown rot on stone fruits, and the disease incidence and disease severity of brown rot were significantly reduced when the fermentation broth of strain ZL261 was applied on peaches. This is the first report of the application of Collimonas sp. to control M. fructicola in China. Our results suggested that strain ZL261 could be a potential biocontrol agent against brown rot on stone fruits caused by M. fructicola. Further work is required to determine the effective control characteristics on a large scale and the suitable dosage for use as a commercial product, including the effect of the proper application of strain ZL261 alone or in combination with another biocontrol agent or in combination with other alternative treatments such as heat treatment, physical treatments, and natural substance treatments.

Compliance and ethics The author(s) declare that they have no conflict of interest.

Acknowledgements This work was supported by funding for training talents in Beijing City (2014000020060G180) to Huiling Wu, the Science and Technology Plan Project of Beijing (D151100003915003) to Weicheng Liu, and the Science and Technology Innovation Fund from the Beijing Academy of Agriculture and Forestry Sciences (QNJJ201416, QNJJ201411) to Jiewei Zhang and Huiling $W u$.

De Boer, W., Klein Gunnewiek, P.J.A., Lafeber, P., Janse, J.D., Spit, B.E., and Woldendorp, J.W. (1998). Anti-fungal properties of chitinolytic dune soil bacteria. Soil Biol Biochem 30, 193-203.

Garbeva, P., Hordijk, C., Gerards, S., and de Boer, W. (2014). Volatiles produced by the mycophagous soil bacterium Collimonas. FEMS Microbiol Ecol 87, 639-649.

Kamilova, F., Leveau, J.H.J., and Lugtenberg, B. (2007). Collimonas fungivorans, an unpredicted in vitro but efficient in vivo biocontrol agent for the suppression of tomato foot and root rot. Environ Microbiol 9, 1597-1603.

Leveau, J.H.J., Uroz, S., and de Boer, W. (2010). The bacterial genus Collimonas : mycophagy, weathering and other adaptive solutions to life in oligotrophic soil environments. Environ Microbiol 12, 281-292.

Pellegrino, C., Gullino, M.L., Garibaldi, A., and Spadaro, D. (2009). First report of brown rot of stone fruit caused by Monilinia fructicola in Italy. Plant Dis 93, 668-668.

Open Access This article is distributed under the terms of the Creative Commons Attribution License which permits any use, distribution, and reproduction in any medium, provided the original author(s) and source are credited.

\section{SUPPORTING INFORMATION}

Figure S1 Phylogenetic tree based on the 16S rDNA sequences, constructed using a neighbor-joining method. The values of the nodes indicate the bootstrap and posterior probability support.

Figure S2 PDA dual culture plates showing antagonistic activities against $M$. fructicola.

Figure S3 Control effects of strain ZL261 on the spore germination of M. fructicola.

Table S1 Comparison of carbon source utilization between strain ZL261 and C. pratensis CCUG 54728 ${ }^{\mathrm{T}}$, C. fungivorans CCUG $48868^{\mathrm{T}}$, and C. arenae CCUG $54727^{\mathrm{T}}$

Table S2 Antagonistic activities of Collimonas sp. strains against $M$. fructicola when co-cultured with $M$. fructicola on PDA dual culture plates at $25^{\circ} \mathrm{C}$ for 7 days

The supporting information is available online at life.scichina.com and link.springer.com. The supporting materials are published as submitted, without typesetting or editing. The responsibility for scientific accuracy and content remains entirely with the authors. 